\title{
Multipoint spacecraft observations of long-lasting poloidal Pc4 pulsations in the dayside magnetosphere on 1-2 May 2014
}

\author{
Galina Korotova $^{1,2}$, David Sibeck ${ }^{3}$, Mark Engebretson ${ }^{4}$, John Wygant $^{5}$, Scott Thaller ${ }^{5}$, Harlan Spence $^{6}$, \\ Craig Kletzing ${ }^{7}$, Vassilis Angelopoulos ${ }^{8}$, and Robert Redmon ${ }^{9}$ \\ ${ }^{1}$ IPST, University of Maryland, College Park, MD, USA \\ ${ }^{2}$ IZMIRAN, Russian Academy of Sciences, Moscow, Troitsk, Russia \\ ${ }^{3}$ Code 674, NASA/GSFC, Greenbelt, MD, USA \\ ${ }^{4}$ Department of Physics, Augsburg College, Minneapolis, MN, USA \\ ${ }^{5}$ College of Science and Engineering, University of Minnesota, Minneapolis, MN, USA \\ ${ }^{6}$ EOS, University of New Hampshire, Durham, NH, USA \\ ${ }^{7}$ Department of Physics and Astronomy, Iowa University, Iowa City, IA, USA \\ ${ }^{8}$ Department of Earth, Planetary and Space sciences, UCLA, Los Angeles, CA, USA \\ ${ }^{9}$ Solar and Terrestrial Physics division, NGDC/NOAA, Boulder, CO, USA
}

Correspondence to: Galina Korotova (gkorotov@umd.edu)

Received: 23 July 2016 - Revised: 19 October 2016 - Accepted: 20 October 2016 - Published: 14 November 2016

\begin{abstract}
We use magnetic field and plasma observations from the Van Allen Probes, Time History of Events and Macroscale Interactions during Substorms (THEMIS) and Geostationary Operational Environmental Satellite system (GOES) spacecraft to study the spatial and temporal characteristics of long-lasting poloidal Pc4 pulsations in the dayside magnetosphere. The pulsations were observed after the main phase of a moderate storm during low geomagnetic activity. The pulsations occurred during various interplanetary conditions and the solar wind parameters do not seem to control the occurrence of the pulsations. The most striking feature of the Pc4 magnetic field pulsations was their occurrence at similar locations during three of four successive orbits. We used this information to study the latitudinal nodal structure of the pulsations and demonstrated that the latitudinal extent of the magnetic field pulsations did not exceed 2 Earth radii $\left(R_{\mathrm{E}}\right)$. A phase shift between the azimuthal and radial components of the electric and magnetic fields was observed from $Z_{\mathrm{SM}}=0.30 R_{\mathrm{E}}$ to $Z_{\mathrm{SM}}=-0.16 R_{\mathrm{E}}$. We used magnetic and electric field data from Van Allen Probes to determine the structure of ULF waves. We showed that the Pc4 magnetic field pulsations were radially polarized and are the secondmode harmonic waves. We suggest that the spacecraft were near a magnetic field null during the second orbit when they failed to observe the magnetic field pulsations at the local
\end{abstract}

times where pulsations were observed on previous and successive orbits. We investigated the spectral structure of the Pc4 pulsations. Each spacecraft observed a decrease of the dominant period as it moved to a smaller $L$ shell (stronger magnetic field strength). We demonstrated that higher frequencies occurred at times and locations where Alfvén velocities were greater, i.e., on Orbit 1. There is some evidence that the periods of the pulsations increased during the plasmasphere refilling following the storm.

Keywords. Magnetospheric physics (solar-windmagnetosphere interactions) - radio science (magnetospheric physics; waves in plasma)

\section{Introduction}

Poloidal Pc4 pulsations $(7 \mathrm{mHz}<f<22 \mathrm{mHz}$ ) characterized by strong displacements of the magnetic field lines in the radial direction are common in both the dayside and nightside magnetosphere (e.g., Hughes et al., 1978). These poloidal pulsations have been attributed to a number of mechanisms, most particularly to field line resonances (Cramm et al., 2000), drift-bounce resonances, ballooning modes and drift mirror mode instabilities (e.g., Engebretson et al., 1992). 
First, consider the location where they occur. Information on the morphology of such waves until recent times was mostly based on geosynchronous and Active Magnetospheric Particle Tracer Explorers Charge Composition Explorer (AMPTE CCE) observations. According to Kokubun et al. (1989) radially polarized Pc4 pulsations occur at all local times but are almost never observed on the ground, the exception being Pg giant pulsations. Radial Pc4 pulsations occur most often in the afternoon during intervals of low solar wind velocity, under quiet geomagnetic conditions of $(\mathrm{AE}<200 \mathrm{nT})$, and exhibit a strong preference for a northward interplanetary magnetic field $\left(B_{z}>0\right)$. Arthur and McPherron (1981) noted that the occurrence of radially polarized Pc4 pulsations at synchronous orbit peaks sharply near dusk, with nearly all events occurring between 11:00 and 23:00 UT. They showed that radially polarized pulsations exhibit a nearly uniform distribution of frequencies over the Pc4 band during daytime and do not show any evident dependence of frequency on local time. Takahashi et al. (1985) used four geosynchronous satellites to show that the frequency distribution for radially polarized Pc4 pulsations was much more spread out than that for pulsations with other polarizations, scattered from 2 to $19 \mathrm{mHz}$ with a mean of $9 \mathrm{mHz}$. Anderson et al. (1990) found that the occurrence distribution of radial waves shows a shift from $L<7$ on the dayside to $L>7$ at midnight.

Hughes et al. (1978) noted that waves with large radial magnetic field components $(\sim 9 \mathrm{nT})$ were rarely seen coherently on two spacecraft indicating a large azimuthal number $(m=100)$. Hughes et al. (1979) described a compressional Pc4 pulsation observed by three geostationary satellites near local midnight and estimated the radial displacement of the plasma to be $0.2 R_{\mathrm{E}}$. Singer et al. (1982) showed that the resonance width of three dayside Pc4-5 pulsation events between $L=4$ and 7 and within $10^{\circ}$ of the geomagnetic equator ranged from 0.2 to 1.6 in $L$ shell and that the two events were observed as the spacecraft encountered the magnetopause.

Anderson et al. (1991) showed that radial pulsations occurred preferentially for cone angles above $60^{\circ}$ and there was a large increase in $\mathrm{AE}$ from 5 to $15 \mathrm{~h}$ before the onset of strong events and a shift from IMF $B_{z}<0$ to $B_{z}>0$ from 2 to $10 \mathrm{~h}$ before the events. Anderson et al. (1990) suggested that the generation of radial pulsations might be associated not with freshly energized particles injected during substorms but with inhomogeneities resulting from the evolution of the previously injected ion distributions that could remain stable for a long time. Anderson et al. (1991) noted that sufficiently low convection electric fields during lower magnetic activity were favorable for energy conversion from particles to waves.

Engebretson et al. (1992) found no cases for which the radially polarized Pc4 pulsations coincided with a sharp plasmapause boundary. They were observed far outside the $L$ shell region where density reached $400 \mathrm{~cm}^{-3}$. Based on the fact that pulsation onsets often occurred shortly after
AE dropped to very low values, they speculated that some property of the low-energy or thermal plasma, or the magnetospheric configuration associated with rapid expansion of the outer plasmasphere during lower magnetic activity, might be the trigger of wave onset in localized regions. They suggested that increased densities of thermal plasma associated with the refilling of the outer plasmasphere might destabilize the marginally stable local ring current population and cause some of the free energy of the hot trapped ions to be transferred to ULF waves via the drift-Alfvén-mirrormode instability. They noted that an examination of plasma in the regions of the generation of $\mathrm{Pc} 4$ pulsations to determine whether it is in a "special conditions with local properties" appears very important. Schäfer et al. $(2007,2008)$ reported that sometimes high-m Pc4 waves were actually seen near the plasmapause and suggested that they may be due to harmonics of poloidal mode eigen oscillations in a radially confined Alfvén resonance region at the inner or outer edges of the plasmapause. Dai et al. (2015) noted that the effect of the plasmapause on the radial transmission of the poloidal waves is caused by the fact that the Alfvén speed changes rapidly across the radial direction.

Eriksson et al. (2005) reported three cases of poloidal Pc5 pulsations with large azimuthal number $(m=100)$ waves observed by Cluster that occurred in the dayside magnetosphere during magnetically quiet times from $L=4$ to $L=6$. They studied observations from LANL satellites for signs of proton injection prior to, or during the observed ULF events but none of the events showed any sign of enhanced proton flux. They ruled out the drift-bounce resonance that would involve unstable particle populations and drift-mirror instability that would require an unreasonably high plasma pressure for the instability criteria to be satisfied and left open the question regarding their generation mechanisms.

Not much is known about long-duration Pc4 pulsations observed in space. However, Rostoker et al. (1979) reported ground magnetometer observations of highly monochromatic Pc4 giant pulsations in the auroral zone that reoccurred on successive days during quiet geomagnetic conditions. They suggested that the Pc4 pulsations may be due to the occurrence of field line resonances at the plasmapause in the region where the electric field changes its azimuthal direction from westward to eastward. Several papers reported observations of long lasting and longer period Pc5 pulsations in the dayside magnetosphere during low magnetic activity (Higbie et al., 1982; Takahashi et al., 1985; Nagano and Araki, 1983; Sarris et al., 2007). Higbie et al. (1982) reported large-amplitude Pc5 waves seen in the magnetic field and particle fluxes that were observed almost continuously for $48 \mathrm{~h}$ by several spacecraft in or near geostationary orbit during the dayside portion of their orbit. They discussed some candidate mechanisms for producing these oscillations such as the Kelvin-Helmholtz instability, global oscillations of the magnetosphere and the magnetospheric convection electric field as an external source of energy but gave no definite 
explanation for this event. Nagano and Araki (1983) suggested that these long-duration Pc5 pulsations may be generated by an instability associated with the plasmapause or the ring current during the recovery phase of a large magnetic storm. Takahashi et al. (1987) studied characteristics of the Pc5 magnetic pulsations initially designated as global Pc5 by Higbie et al. (1982) and concluded that such waves are likely to be excited by an internal instability associated with the ring current, possibly the drift mirror instability. Sarris et al. (2007) studied a long-duration Pc5 event observed for more than 2 days at geosynchronous orbit over several traversals of the two Geostationary Operational Environmental Satellite system (GOES) satellites. They suggested generation mechanisms but like previous authors did not provide conclusive evidence for that.

NASA's Van Allen Probe mission, which carries the most comprehensive instrumentation ever sent to the radiation belts, provides new opportunities to study wave-particle interactions. Two recent case studies (Dai et al., 2013; Claudepierre et al., 2013) employed Van Allen Probe observations to describe the drift resonant interactions of poloidal Pc4 and 5 waves with electrons and ions. Korotova et al. (2015) presented observations of poloidal Pc4 pulsations with limited azimuthal extent seen by the Van Allen Probe B spacecraft in the pre-midnight inner magnetosphere and showed that the most likely source of this wave was a bounce resonance interaction of second-harmonic waves with the protons. That was in agreement with many studies that determined that poloidal Pc4 pulsations are second-harmonic Alfvén waves resonant along a magnetic flux tube (e.g., Cummings et al., 1969; Singer et al., 1982; Takahashi and McPherron, 1984). Motoba et al. (2015) reported one-to-one associations of these pulsations with auroral pulsations around the ionospheric footprint of the magnetic field line and suggested that an auroral undulation event is closely linked to the generation of second-harmonic poloidal waves. Dai et al. (2015) presented a comprehensive statistical study of poloidal Pc4 pulsations observed by Van Allen Probes spacecraft. To get more information on the free-energy wave source for the inner magnetosphere they sorted the pulsations into compressional and non-compressional waves based on the ratio of radial $\left(B_{\mathrm{r}}\right)$ to compressional $\left(B_{\mathrm{p}}\right)$ components. They believe that waves with a small (large) compressional component correspond to an internal (external) wave source. They found that the compressional Pc4 poloidal waves have their origin in the solar wind, while the non-compressional waves can be attributed to the decay of the ring current in the late storm recovery phases. They found that the energy source for non-compressional Pc4 poloidal waves was associated with the decay of the ring current in the late recovery phase of geomagnetic storms, while the compressional Pc4 poloidal waves were associated with variations of solar wind dynamic pressure in geomagnetic storms.

Despite many papers devoted to poloidal Pc4 pulsations, information about their nodal structure, their mode, latitudi- nal and radial extents remains rather limited. Yet such information is needed to clarify the energy source for their generation. We present a new case study of long-lasting strong poloidal Pc4 pulsations observed by multiple spacecraft during three of four successive orbits in the dayside magnetosphere from 00:00 UT on 1 May 2014 to 12:00 UT on 2 May 2014. We wish to determine whether solar wind parameters control the occurrence and recurrence of these pulsations. We then investigate whether pulsations are observed in close proximity to the magnetopause and whether the plasmapause has an effect on generation of the pulsations. We also wish to find the frequency of the pulsations as a function of radial distance from Earth. Though no obvious cause for their generation was found, the study of their spatial and temporal characteristics helps us understand the conditions that are favorable for triggering these pulsations.

\section{Instruments and spacecraft orbit locations}

The two Van Allen Probes were launched in August 2012 into nearly identical equatorial and low-inclination $\left(\sim 10^{\circ}\right)$ orbits with perigee altitudes of 605 and $625 \mathrm{~km}$ and apogees of 30410 and $30540 \mathrm{~km}$ (Mauk et al., 2012). Both satellites carry identical sets of instruments to measure charged particle populations, fields and waves in the inner magnetosphere. The observations reported here consist of combined measurements of the Electric and Magnetic Field Instrument Suite and Integrated Science (EMFISIS) (Kletzing et al., 2013) and Electric Field and Waves Suite (EFW) instruments (Wygant et al., 2013). We used the magnetic field data with $4 \mathrm{~s}$ time resolution and electric field data with $\sim 11 \mathrm{~s}$ time resolution. Additionally we used magnetic field data with $3 \mathrm{~s}$ time resolution data from the Time History of Events and Macroscale Interactions during Substorms (THEMIS) triaxial fluxgate magnetometers (FGMs; Auster et al., 2008). We used magnetic field data from GOES 15 with $0.5 \mathrm{~s}$ time resolution (Singer et al., 1996). We used Wind solar wind magnetic field and plasma data with $3 \mathrm{~s}$ (Lepping et al., 1995) and $1 \mathrm{~min}$ time resolution, respectively (Ogilvie et al., 1995). Figure 1 shows the orbits of Van Allen Probes A, B and THEMIS $\mathrm{D}$ and $\mathrm{E}$ in GSM coordinates from 00:00 UT on 1 May 2014 to $12: 00 \mathrm{UT}$ on 2 May 2014. They move from GSM ( $X, Y$, $Z)=(3.64,0.78,-0.32) R_{\mathrm{E}}$, from $\operatorname{GSM}(X, Y, Z)=(-0.3$, $1.1,0.41) R_{\mathrm{E}}$, from GSM $(X, Y, Z)=(9.13,-4.67,-2.79)$ $R_{\mathrm{E}}$ and from $\operatorname{GSM}(X, Y, Z)=(6.49,-7.39,-3.47) R_{\mathrm{E}}$, respectively. Note the broad range in GSM $Z$ values covered at apogee for Van Allen Probes spacecraft.

\section{Observations}

\subsection{Solar wind and geomagnetic conditions}

Figure 2 shows the Wind magnetic field and plasma observations from 01:00 UT on 1 May 2014 to 10:00 UT on 2 May 2014 when it moved from GSM $(X, Y, Z)=(254.62$, 

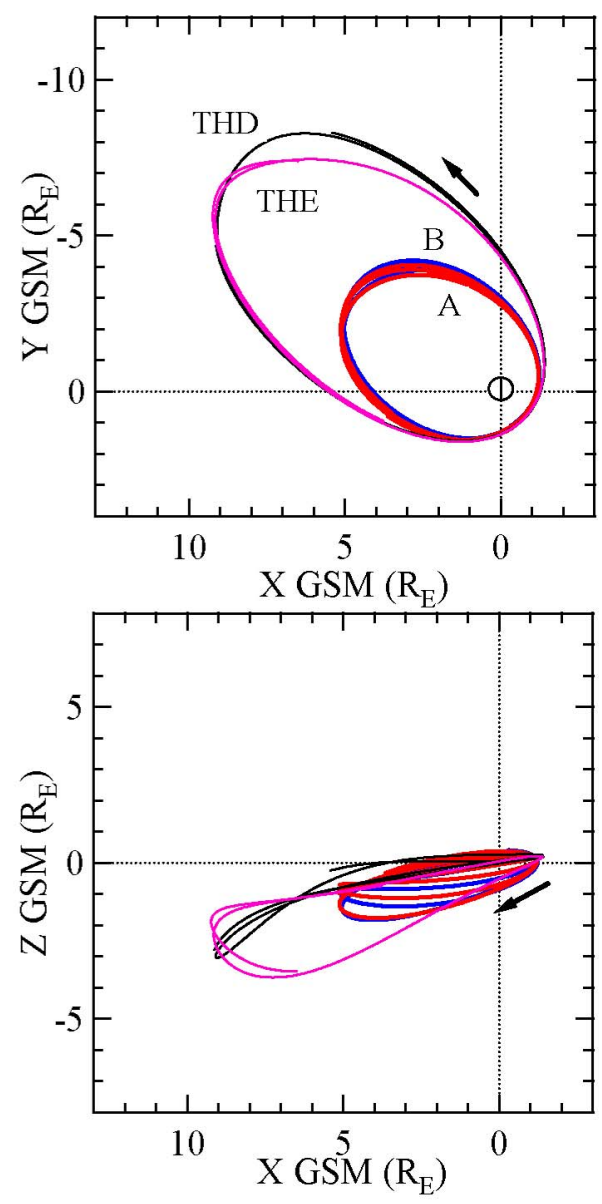

Figure 1. GSM Locations of Van Allen Probes A (in red line) and $\mathrm{B}$ (blue line) and THEMIS D (THD; black line) and E (THE; pink line) for the selected interval from 00:00 UT on 1 May 2014 to 12:00 UT on 2 May 2014.

$-60.48,-10.96) R_{\mathrm{E}}$ to $\operatorname{GSM}(X, Y, Z)=(253.26,-64.27$, 10.20) $R_{\mathrm{E}}$. Lag times from Wind to the Earth are in the range of 90 to $70 \mathrm{~min}$ for the observed velocities from 300 to $380 \mathrm{~km} \mathrm{~s}^{-1}$. Solid red bars show time-shifted intervals when Pc4 magnetic field pulsations were observed by multiple spacecraft in the dayside magnetosphere. Dashed red bars indicate times when the $\mathrm{Pc} 4$ pulsations were observed in the Van Allen Probe electric field, not in the magnetic field. During the four Van Allen Probe orbits the pulsations occurred for various IMF conditions and the solar wind parameters do not seem to control the occurrence of the pulsations. The magnetic field strength, cone angle and density of the solar wind could be as high as $10.4 \mathrm{nT}, 98^{\circ}$ and $13 \mathrm{~cm}^{-3}$ and as low as $2.3 \mathrm{nT}, 20^{\circ}$ and $3.2 \mathrm{~cm}^{-3}$, respectively. As far as the other solar wind parameters are concerned, during each of the four intervals marked by solid and dashed red bars, the most common conditions for the generation of the pulsations are spiral IMF orientations $\left(B_{x}<0, B_{y}>0\right), B_{z}>0$ and low dynamic pressure not exceeding $2.5 \mathrm{nPa}$. Figure 3 shows geomagnetic indices from 12:00 UT on 29 April to 11:00 UT on 2 May 2014. Solid and dashed bars indicate the intervals of Pc4 pulsations observed on 1-2 May 2014. A moderate storm was observed on 30 April 2014 in which the Dst index reached a maximum negative value of $-64 \mathrm{nT}$ at 09:30 UT. The storm lasted more than $20 \mathrm{~h}$ and resulted in enhanced geomagnetic activity characterized by the development of an intense electrojet with the AE index exceeding $1200 \mathrm{nT}$. The pulsations occurred after the main phase of the moderate storm during low geomagnetic activity with $\mathrm{Kp}<3$ and $\mathrm{AE}<200 \mathrm{nT}$. A high occurrence of Pc4 poloidal pulsations in the storm recovery phase has been reported by Dai et al. (2015).

\subsection{Van Allen Probe A and B observations}

The magnetic field pulsations occurred at similar locations on three of four successive orbits plotted in Fig. 4 in red and blue for Van Allen Probes A and B, respectively. Each orbit is $9 \mathrm{~h}$ apart from 00:00 UT on 1 May 2014 to 12:00 UT on 2 May 2014; the thick red and blue line segments in Fig. 4 show the locations of Van Allen Probes A and B where the Pc4 magnetic field pulsations occurred on orbits 1, 3 and 4 . To determine the type and harmonic mode of the waves we transformed the GSE magnetic field observations of the Pc4 pulsations into field-aligned coordinates and present them in Figs. 5a-c. In this coordinate system the $Z$ axis is in the direction of the averaged magnetic field. The $Y$ axis is transverse to $\mathrm{B}$ and to the radius vector from the Earth to the spacecraft and is directed approximately azimuthally eastward. The $X$ axis completes the right-handed system and is directed approximately radially outward from Earth. The pulsations are poloidal with strong radial $\left(B_{x}\right)$ magnetic field perturbations. They occurred from $L=4.3$ to 5.5 and exhibited packet-like structures with peak-to-peak amplitudes ranging from 1 to $12 \mathrm{nT}$. They were observed at local times from $\sim$ 07:30 to 11:30 MLT with maximum amplitudes occurring from $\sim 09: 30$ to 11:00 MLT. Their periods ranged from 35 to $120 \mathrm{~s}$ and the duration of individual events did not exceed $3.5 \mathrm{~h}$.

Simultaneous measurements of the electric and magnetic fields provide us with unique opportunities to study theoretical properties of the pulsations. To determine the most important property, namely the harmonic mode of the waves, we use the approach of Takahashi et al. (2013), who considered the phase relationship between the azimuthal component of the electric field $\left(E_{y}\right)$ and the radial component of the magnetic field $\left(B_{x}\right)$ relative to the equator. We obtained the three-dimensional Van Allen Probes electric field by assuming $\boldsymbol{E} \cdot \boldsymbol{B}=0$. Figure 6 shows the phase relationship between $B_{x}$ and $E_{y}$ for Van Allen Probes A and B on orbits 1 and 3 when the spacecraft were south of the equator. The amplitude of $E_{y}$ presented in Figs. 6 and 7 was multiplied by a factor of 2 to better display the visual effects. $E_{y}$ lags $B_{x}$ by $90^{\circ}$, clearly indicating that the Pc4 pulsations are 


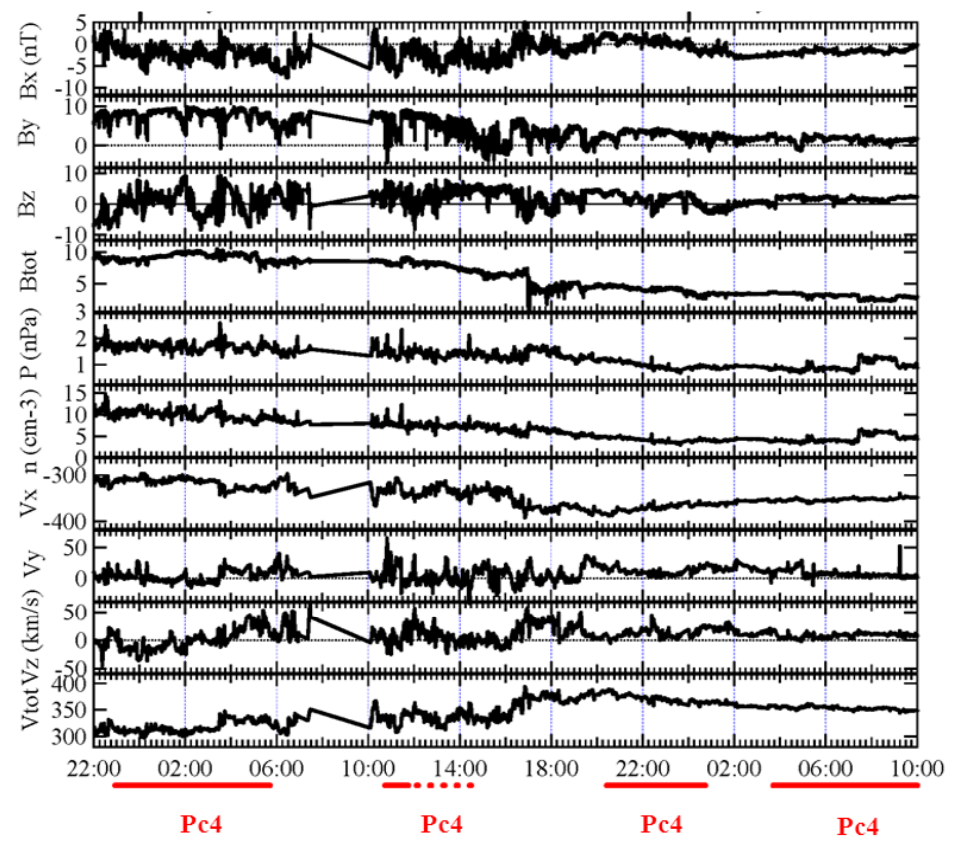

Figure 2. Wind plasma and magnetic field observations in GSM coordinates from 22:00:00 UT on 30 April 2014 to 10:00 UT on 2 May 2014. Solid red bars show time-shifted intervals when Pc4 magnetic field pulsations were observed by multiple spacecraft. Dashed red bars indicate times when the Pc4 pulsations were observed in the electric field, not in the magnetic field. (See text in Sect. 3.4).

second-harmonic mode waves during these orbits. The magnetic equator is the location of the antinode (maximum amplitude) of field line displacement and the node of magnetic field perturbations for the fundamental mode, whereas it is the location of the node (zero amplitude) of field line displacement and antinode of magnetic field perturbations for the second harmonic (Southwood and Kivelson, 1982). The range of periods of the observed $\mathrm{Pc} 4$ pulsations fits that predicted for second-harmonic waves in several previous theoretical papers (e.g., Cummings et al., 1969). Large magnetic field oscillations in the $B_{x}$ component and small electric field oscillations in the $E_{y}$ component near the magnetic equator also confirm the second-harmonic mode structure. Our results are in agreement with numerous observations indicating that poloidal waves are excited mostly at the second harmonic (e.g., Hughes et al., 1978). Southwood (1976) explained the preferential excitation of the second-harmonic mode in the ring current environment.

Figure 7 shows how the phase relations (left panels) on Orbit 4 depend on the locations of the spacecraft relative to the equator (right panels). During the first (third) intervals $E_{y}$, lags (leads) $B_{x}$ by $90^{\circ}$ when the spacecraft were south (north) of the equator, in agreement with our previous finding that the pulsations are the second-harmonic waves. Observations during the second intervals when the spacecraft crossed the equator indicate mixed-phase relationships and double-frequency pulsations (Korotova et al., 2013). The mixed-phase shift between the azimuthal and radial components of the electric and magnetic fields was observed from
$Z_{\mathrm{SM}}=0.30 R_{\mathrm{E}}$ to $Z_{\mathrm{SM}}=-0.16 R_{\mathrm{E}}$. When Van Allen Probe B moved up to $R_{\mathrm{E}}=0.34$, negative lags dominated but were not observed during the whole third interval.

\subsection{Spatial characteristics of Pc4 pulsations}

To study the spatial characteristics of the Pc4 pulsations on 1-2 May 2014 we examined the magnetic field observations from multiple spacecraft during the four corresponding orbits and found that THEMIS E and D and GOES $15 \mathrm{ob}-$ served similar pulsations. The green, purple and black lines in Fig. 4 mark the locations where Pc4 magnetic field pulsations were observed by THEMIS D, THEMIS E and GOES 15 , respectively, during the four corresponding orbits. The region where pulsations occurred lies from $L$ of 5 to 8 and extended to local evening hours. The Pc4 pulsations observed by THEMIS D and GOES 15 during the first (upper panel) and third (bottom panel) orbits are presented in Fig. 5d and e, respectively. Figure 5e shows the GOES 15 poloidal pulsations observed in the afternoon-evening sector. They exhibited maximum amplitudes of 6-9 nT, and their periods changed from 60 to $83 \mathrm{~s}$ during the orbits. The similarity of the Pc4 pulsations observed by the Van Allen Probe A and GOES 15 is seen in Fig. 5f where the radial components of their magnetic fields are presented from 00:40 to 01:30 UT on 2 May 2014 during Orbit 3. We believe that they are produced by the same source due to similar periods and amplitudes (component by component). 


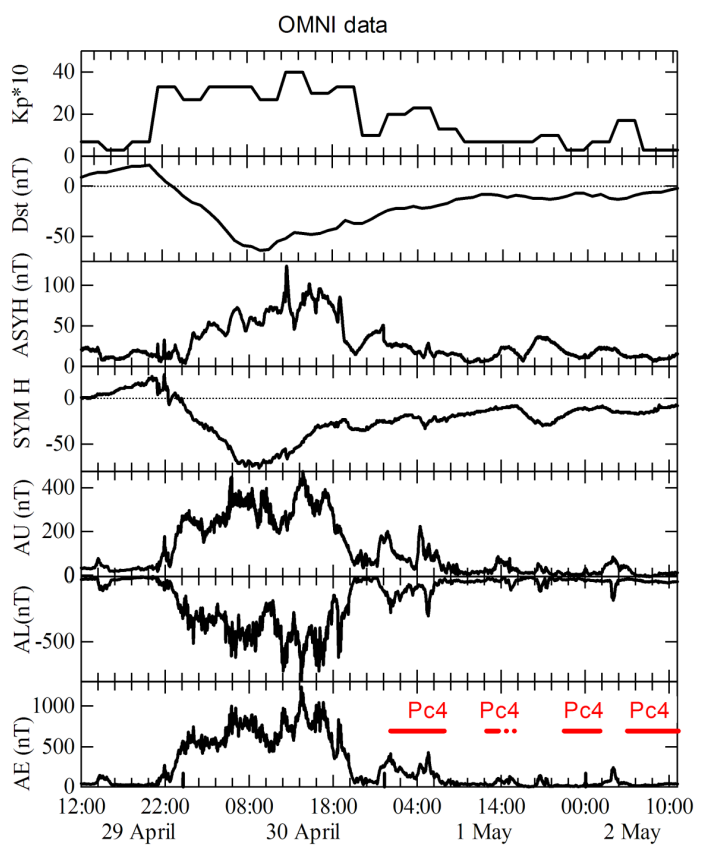

Figure 3. Kp, Dst, ring current and electrojet intensity indices from 12:00 UT on 29 April 2014 to 11:00 UT on 2 May 2014 available from the OMNI database (http://omniweb.gsfc.nasa.gov/) . Solid red bars show intervals when Pc4 magnetic field pulsations were observed by multiple spacecraft. Dashed red bars indicate times when the Pc4 pulsations were observed in the electric field, not in the magnetic field.

The location of THEMIS E during the second orbit is indicated by a dotted line in Fig. 4 because the amplitude of the pulsations was very small $(<1 \mathrm{nT})$.

\subsection{Nodal latitudinal structure of waves}

To study the latitudinal structure of the pulsations, Fig. 8 presents four successive Van Allen Probe A and B orbits (plotted in red and blue, respectively) shown in the SM $X-Z$ plane from 00:00 UT on 1 May 2014 to 12:00 UT on 2 May 2014. The magnetic pulsations were seen on orbits 1,3 and 4 at locations near the geomagnetic equator, but not on Orbit 2. Since the spacecraft were at similar radial distances and local times on Orbit 2, we infer that the difference lies in the fact that they were near an off-equatorial node near $Z=0.7 R_{\mathrm{E}}$ when at apogee on this orbit. The absence of Pc4 magnetic field pulsations during Van Allen Probe Orbit 2 should not be interpreted as evidence that the pulsations had ceased on Orbit 2. Figure 9 shows that they continued to be present in the electric field observations, more pronounced at Van Allen Probe B. In Figs. 2 and 3 red dashed lines indicate the intervals where the electric field pulsations occurred on Orbit 2. We should note that they were very irregular. Finally, note that the Van Allen Probes spacecraft observed magnetic field pulsations as far south as $Z=-1.1 R_{\mathrm{E}}$. The green, purple and black lines in Fig. 8 indicating locations where THEMIS
D, THEMIS E and GOES 15 observed Pc4 pulsations during corresponding orbits are consistent with an inferred latitudinal extent of at least $2 R_{\mathrm{E}}$.

\subsection{Spectral characteristic of the Pc4 pulsations}

We investigated the spectral structure of the Pc4 pulsations. The overall features of the different waves become more visible in the dynamic spectra display where the power spectral density is defined with respect to the background. Figure 10 shows an example of the dynamic spectra calculated for the radial, azimuthal and compressional components of the Van Allen Probe A magnetic field, during Orbit 3 from 21:30 to $02: 30$ UT on 1-2 May 2014. A time window 128 points $(\sim 8.5 \mathrm{~min})$ long was shifted by 20 points $(\sim 1.3 \mathrm{~min})$ for successive calculations of spectra using the fast Fourier transform (FFT) method. Data at a $4.0 \mathrm{~s}$ cadence were rotated into $5 \mathrm{~min}$ sliding averaged mean field-aligned coordinates, averaged to $8.0 \mathrm{~s}$ and differenced before transforming. Differencing was used in order to facilitate display of a wide range of spectral power by removing the $f^{-2}$ fall off in spectral power with frequency (Engebretson et al., 2015). Power at frequencies from 0 to $0.062 \mathrm{~Hz}$ in each of these three orthogonal components is color-coded according to the color bar shown on the right. Beneath these three panels are listed the universal time (UT), MLT, MLAT and $L$ shell. The magnetic field dynamic spectra exhibit several broad frequency band enhancements of pulsations from 23:00 to 01:30 UT that coincide with the intervals of large pulsation amplitudes of the pulsations seen in the original data. The constant tone at $\sim 31 \mathrm{mHz}$ is an instrumental effect. However, the narrow band frequency enhancement between 10 and $25 \mathrm{mHz}$ from 23:07 to 01:00 UT is real. It is evident in all three components, but the strongest spectral power density occurs in the radial $B_{x}$ component, corresponding to the second-mode harmonic of the poloidal waves. Only weak spectral features are seen in the compressional $B_{z}$ component. Some pulsations observed during the three orbits might be categorized as compressional ones based on the classification of Dai et al. (2015). They consider pulsations to be compressional for even very weak $B_{\mathrm{p}}$, i.e., for abs $\left(B_{\mathrm{p}} / B_{\mathrm{r}}\right)>0.2$. But we consider them to be non-compressional for several reasons. They exhibit weak amplitudes in the compressional component and have temporal and spatial features like those of the non-compressional pulsations discussed by Dai et al. The strong compressional components observed by THEMIS D on Orbit 1 can be attributed to Pc5 pulsations generated simultaneously with the Pc4 pulsations but having a different source. The absence of ground signatures for the Pc4 pulsations suggests that the observed pulsations have large azimuthal numbers and an internal wave source because ULF waves with small azimuthal wavelengths are reflected from the ionosphere when they propagate from the equatorial regions to the ground. Besides, we ruled out any solar wind drivers, e.g., variations in dynamic pressure. Taking into ac- 
Orbit 1
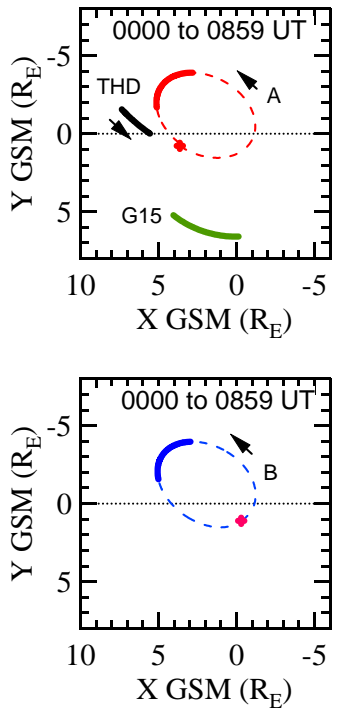

Orbit 2
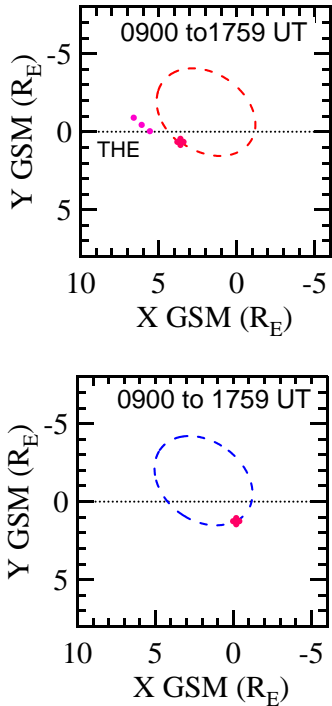

Orbit 3
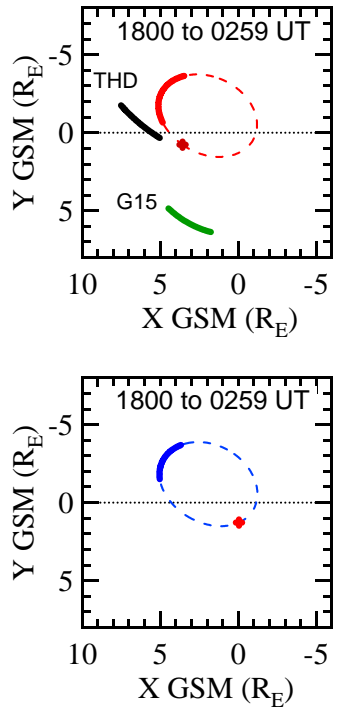

Orbit 4
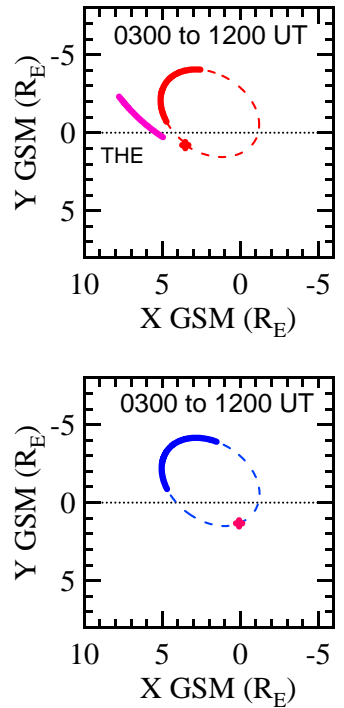

Figure 4. Four successive Van Allen Probe A and B orbits (plotted in red and blue, respectively) in the GSM $X-Z$ plane from 00:00 UT on 1 May 2014 to 12:00 UT on 2 May 2014. The red dots show the start of the corresponding orbits. The thick red and blue line segments show the locations of Van Allen Probes A and B where the Pc4 magnetic field pulsations occurred on orbits 1, 3 and 4. The green, purple and black lines mark the locations where Pc4 magnetic field pulsations were observed by THEMIS D, THEMIS E and GOES 15, respectively, during the four corresponding orbits.

count of all of these factors we believe that the long-lasting poloidal Pc4 pulsations were produced through the driftbounce resonance of tens of kilo-electron-volt ions with the second-harmonic mode waves.

It is obvious that the dominant spectral frequency tends to increase with decreasing $L$. The distinct spectral power density enhancement starts in a narrow frequency band at about $12 \mathrm{mHz}$ at 22:30 UT, goes down to $10 \mathrm{mHz}$ at 23:30 UT, when the satellite was near the apogee $\left(R_{\mathrm{E}}=5.75\right)$, and then goes up again to about $18 \mathrm{mHz}$ in a broader frequency band.

To study the spatial and temporal characteristics of the pulsations, we calculated Fourier power spectra of the magnetic field in field-aligned coordinates for three orbits when the magnetic field pulsations were observed. The data were not differenced to produce these line spectra, but the mean total field calculated at each time step was subtracted from the compressional component before FFT processing. We confirmed that the periods in the spectra depend on orbit and radial distance. Figure 11 presents an example of the spectra computed for the Van Allen Probe A magnetic field observations during two intervals: from 23:00 to 23:30 UT and from 01:00 to 01:30 UT. The dominant frequency peak increased from 12 to $15 \mathrm{mHz}$ (period decreased from 83 to $67 \mathrm{~s}$ ) when the satellite moved from $L_{\mathrm{avr}}=5.75$ during the first interval to $L_{\mathrm{avr}}=5.35$ during the second interval. Figure 12 summarizes results for the dominant periods in the spectra of the radial component of the magnetic field observed by all four satellites as a function of the $30 \mathrm{~min}$ averaged magnetic field strength (a) and $L$ shells (b). Dashed curves represent poly- nomial fits with three terms. Note that the range of periods observed on Orbit 1 is less in comparison with those observed on orbits 3 and 4 . Each spacecraft observes a decrease of the dominant period as it moves to a smaller $L$ (stronger magnetic field strength). On Orbit 1 periods decreased from 76 to $48 \mathrm{~s}$, on Orbit 3 from 149 to $59 \mathrm{~s}$ and on Orbit 4 from 166 to $77 \mathrm{~s}$. A tendency for periods to decrease as the International Sun-Earth Explorer (ISEE)-1 and -2 spacecraft moved inward from $L=6.8$ to $L=4$ through the late morning sector was reported by Singer at el. (1979). They suggested that the radial dependence of the wave period was a spatial rather than a temporal effect.

We included information on the GOES 15 periods in Fig. 12 (the green crosses). The GOES 15 periods for Orbit 1 lie close to the curves defined for THEMIS and Van Allen Probe observations. However, the GOES 15 periods for Orbit 3 do not. Their periods are smaller. We suppose that postnoon densities are lower at the GOES 15 location than at the THEMIS and Van Allen Probe prenoon locations for Orbit 3.

A simple explanation for this behavior of pulsation frequencies with radial distance can be given in terms of standing Alfvén waves along resonant field lines (Sugiura and Wilson, 1964). If the observed frequency is the resonant frequency of the field lines at the satellites, then the approximate time of flight expression for the period of oscillations of a standing wave (Wild et al., 2005) is given by $T=$ integral $\mathrm{d} s / V_{\mathrm{A}}(s)$, where the integral is taken along the magnetic field line, ds is a differential length along the field line, and $V_{\mathrm{A}}$ is the Alfvén speed. Therefore the changing shape of the 
(a)
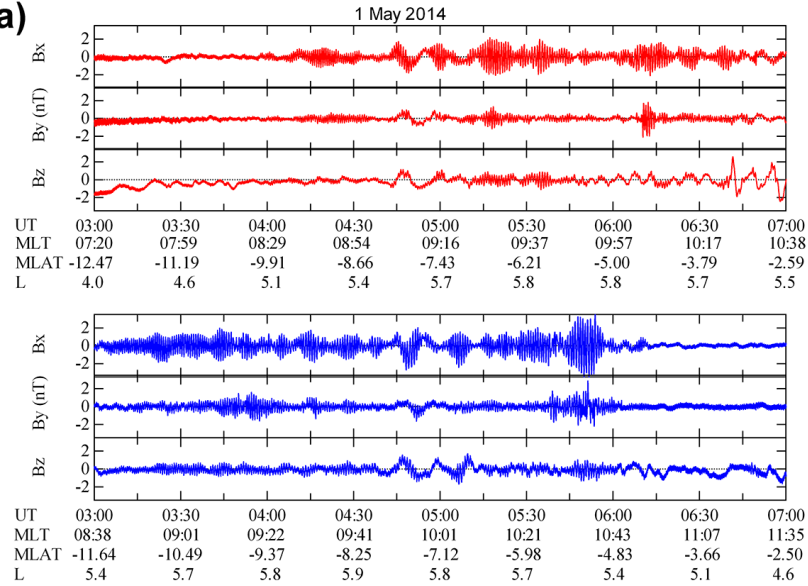

(b)
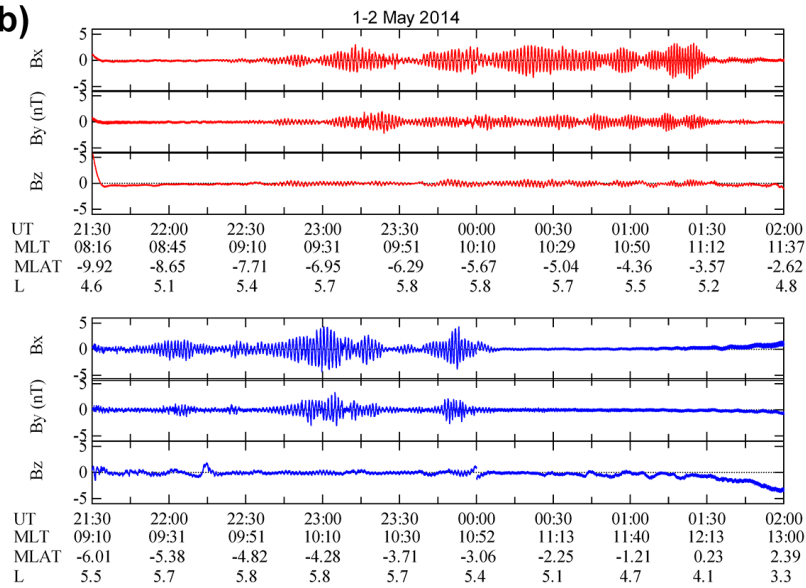

(c)

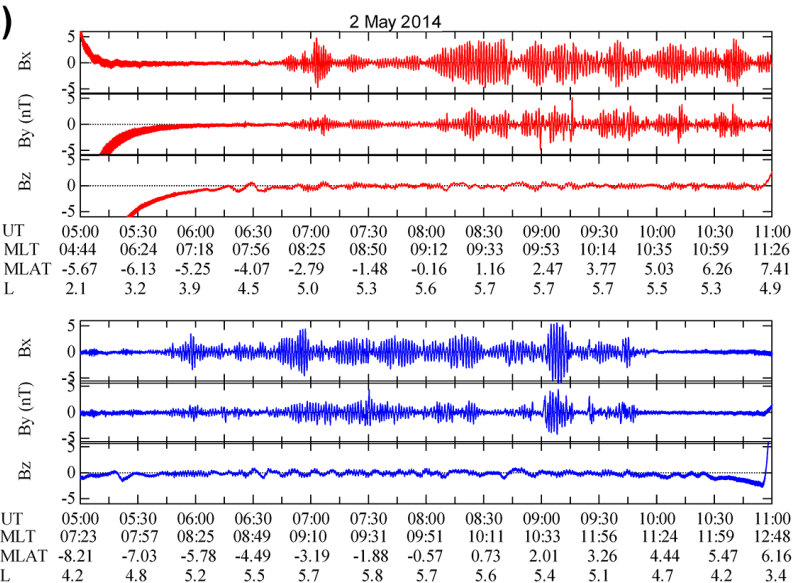

(d)

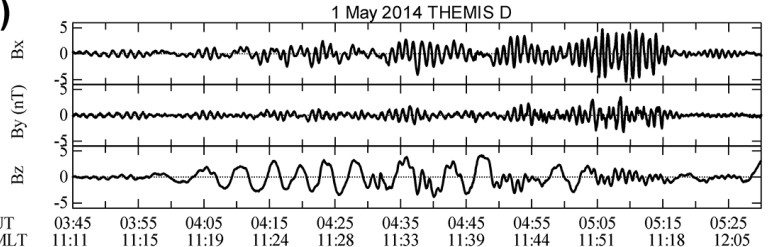

$\begin{array}{llllllllllll}\text { UT } & 03: 45 & 03: 55 & 04: 05 & 04: 15 & 04: 25 & 04: 35 & 04: 45 & 04: 55 & 05: 05 & 05: 15 & 05: 25 \\ \text { MLT } & 11: 11 & 11: 15 & 11: 19 & 11: 24 & 11: 28 & 11: 33 & 11: 39 & 11: 44 & 11: 51 & 11: 18 & 12: 05 \\ \text { MLAT } & -5.42 & -5.30 & -5.17 & -5.03 & -489 & -773 & -4.56 & -38 & -419 & -3.99 & -3.79\end{array}$ $\begin{array}{lccccccccccc}\text { MLAT } & -5.42 & -5.30 & -5.17 & -5.03 & -4.89 & -4.73 & -4.56 & -4.38 & -4.19 & -3.99 & -3.79 \\ \mathrm{~L} & 7.7 & 7.6 & 7.3 & 7.1 & 6.9 & 6.7 & 6.4 & 6.2 & 5.9 & 5.7 & 5.4\end{array}$

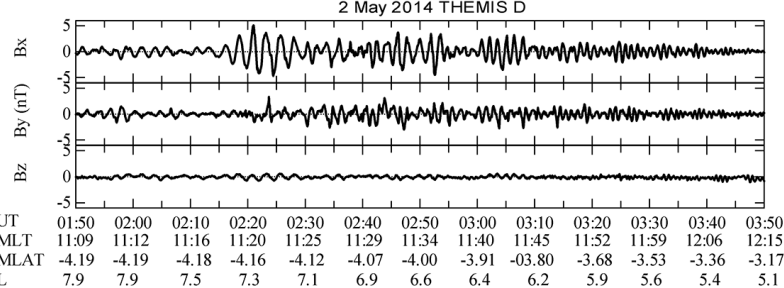

(e)
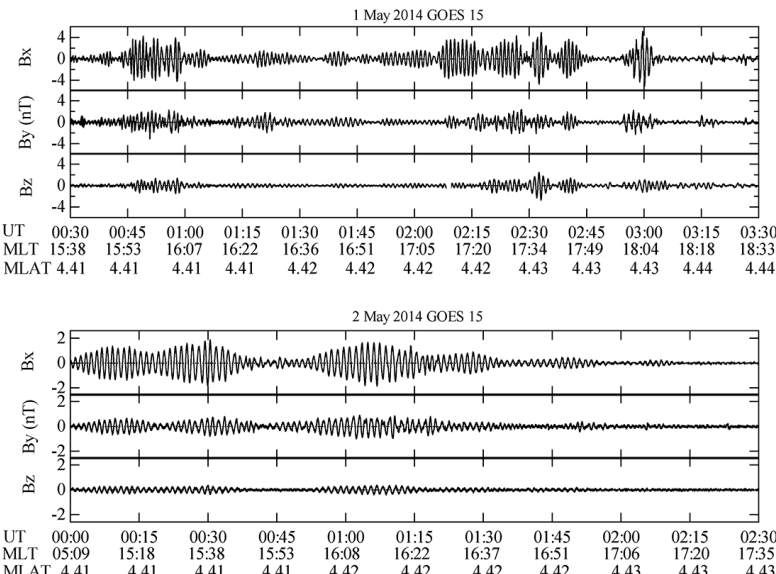

(f)
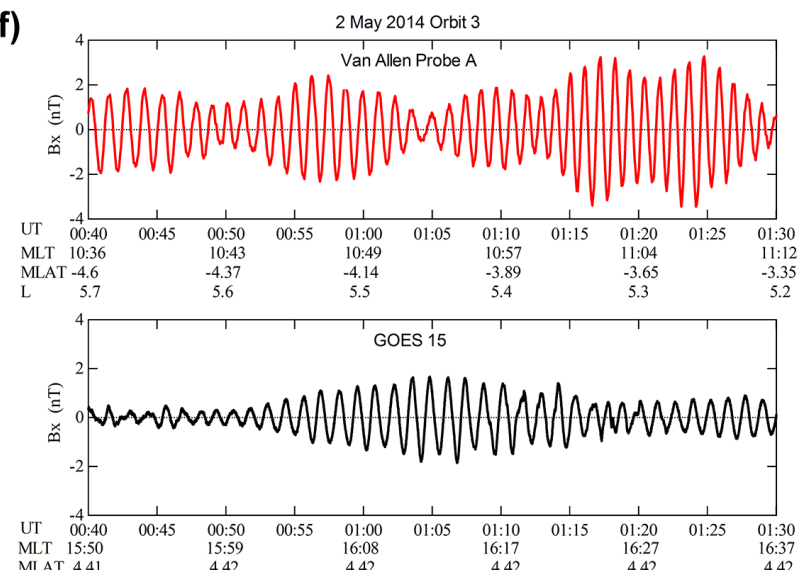

Figure 5. (a) Magnetic field observations in field-aligned coordinates from Van Allen Probes A ( plotted in red) and B (plotted in blue) from 03:00 to 07:00 UT on 1 May 2014 (Orbit 1). The $Z$ axis is in the direction of the averaged magnetic field. The $Y$ axis is transverse to $\mathrm{B}$ and to the radius vector from the Earth to the spacecraft and is directed approximately azimuthally eastward. The $X$ axis completes the right-handed system and is directed approximately radially outward from Earth. The location of the satellite is shown in the bottom where $L$ is the equatorial distance to the field line, MLAT is magnetic latitude in degrees and MLT is the magnetic local time in hours. (b) Magnetic field observations in field-aligned coordinates from Van Allen Probes A and B from 21:30 UT on 1 May 2014 to 02:00 UT on 2 May 2014 (Orbit 3). (c) Magnetic field observations in field-aligned coordinates from Van Allen Probes A and B from 05:00 to 11:00 UT on 2 May 2014 (Orbit 4). (d) Magnetic field observations in field-aligned coordinates from THEMIS D from 03:45 to 05:30 UT on 1 May 2014 (Orbit 1) and from 01:50 to 03:50 UT on 2 May 2014 (Orbit 3). (e) Magnetic field observations in field-aligned coordinates from GOES 15 from 00:30 to 03:30 UT on 1 May 2014 (Orbit 1) and from 00:00 to 02:30 UT on 2 May 2014 (Orbit 3). (f) The radial components of the Pc4 pulsations observed by the Van Allen Probe A and GOES 15 spacecraft from 00:40 to 01:30 UT on 2 May 2014 during Orbit 3. 
(a)
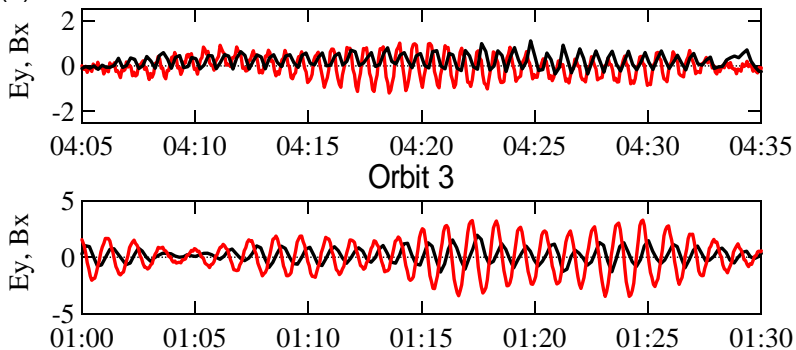

(b)
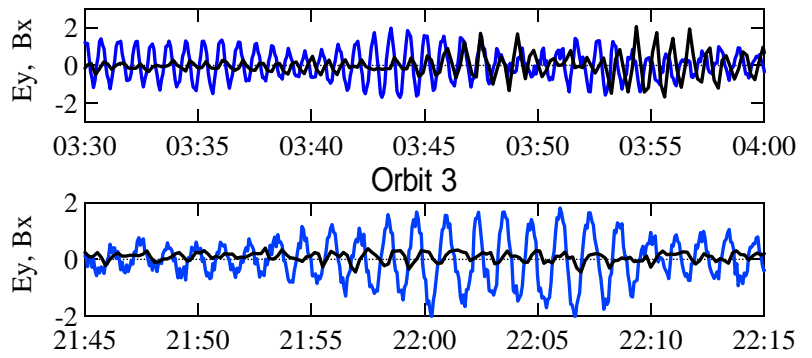

Figure 6. Van Allen Probes A (plotted in red) and B (plotted in blue) observations of electric and magnetic fields on orbits 1 and 3 show that $E_{y}$ (plotted in black) lags $B_{x}$ by $90^{\circ}$ indicating that the Pc4 pulsations are second-harmonic mode poloidal oscillations. $E_{y}$ and $B_{x}$ are displayed in $\mathrm{mV} \mathrm{m}^{-1}$ and $\mathrm{nT}$, respectively.

field lines, the magnetic field strength and the plasma density distribution determining the Alfvén velocity will control the periods of the pulsations. Shorter field line lengths, and enhanced field strengths as the satellite moves to smaller $L$ values that increase the propagation speed of Alfvén pulsation, reduce the pulsation period, in agreement with our results. The continuous decrease of periods of the pulsations with time and $L$ shells is consistent with the locations of the Van Allen Probes being outside the plasmapause during these intervals. Lin et al. (1986) reported a decrease of periods for transverse pulsations in the Pc3-4 frequency range observed by DE-1 spacecraft near the plasmapause with a decrease of $L$ shells and suggested that the each field line resonated at its own eigen period with little interaction between adjacent oscillating shells, and the resonance width was narrow $(<0.1 L)$. They also noted that the eigen period is more sensitive to a change in $L$ than density.

Further understanding of the temporal and spatial factors that govern the occurrence of these waves requires a knowledge of the expected eigen periods of the field lines as a function of magnetic latitude, local time, magnetic activity and season. There are numerous papers (e.g., Orr and Matthew, 1971; Poulter et al., 1988; Takahashi and McPherron, 1984; Schäfer et al., 2008) devoted to theoretical calculations of characteristic periods by solving the wave equations for propagation of low-frequency hydromagnetic waves in plasma under various simplifying assumptions. Cummings et al. (1969) numerically determined the eigen frequencies for the uncoupled toroidal and poloidal modes on a field line at $6.6 R_{\mathrm{E}}$ at the equator for the fundamental through sixth harmonic of the oscillation. Singer et al. (1979) used the Cummings et al. (1969) model in a non-dipolar field geometry with the observed mass densities from ISEE-1 plasma observations to predict periods of fundamental and second-harmonic poloidal waves in the dayside magnetosphere. They found that for the second-harmonic wave, periods ranged from 0.6 to $2.1 \mathrm{~min}$, also consistent with the periods of the Pc4 pulsations presented in our study.

Wild et al. (2005) used a time-of-flight approximation to investigate the latitudinal, diurnal and seasonal variations in the frequency/period of Alfvén pulsations. They found that upstream interplanetary conditions exert a powerful influence upon their period. They noted that an increase in dynamic pressure (from 2 to $10 \mathrm{nPa}$ ) may result in a moderate frequency increase at most magnetic local times, while changes in the Dst parameters may result in large variations in the estimated pulsation frequency (up to a factor of 2 in some cases) in the dayside magnetosphere.

The longer Pc4 pulsation periods observed by the Van Allen Probes on orbits 3 and 4 than on Orbit 1 can be explained by the different solar wind and magnetospheric conditions prevailing on these orbits. For example, the Wind observations presented in Fig. 2 show higher solar wind dynamic pressures and stronger interplanetary magnetic field strengths ( $2 \mathrm{nPa}$ and up to $10.4 \mathrm{nT})$ during Orbit 1 than during orbits 3 and $4(1 \mathrm{nPa}$ and $4 \mathrm{nT})$. To check this hypothesis, we used the magnetic field observations and the plasma densities extracted from Van Allen Probe Electric Field and Waves (EFW) instrument potentials to compute the Alfvén velocities. From top to bottom, Fig. 13 shows densities, total magnetic field strengths and Alfvén velocities vs. universal time for Van Allen Probes A (plotted in red) and B (plotted in blue) from 01:00 UT on 1 May 2014 to 12:00 UT on 2 May 2014. Black bars show intervals when the magnetic field Pc4 pulsations were observed on orbits 1,3 and 4 . Ticks above the panels show spacecraft $L$ values for the corresponding intervals. Alfvén velocities near apogee on orbits 3 and 4 ranged from 300 to $600 \mathrm{~km} \mathrm{~s}^{-1}$, but from 900 to $1500 \mathrm{~km} \mathrm{~s}^{-1}$ on Orbit 1. Since magnetospheric magnetic field strengths this deep in the magnetosphere do not vary greatly (see middle panels A and B), the different Alfvén velocities result primarily from changes in the plasma density (see upper panels A and B). Indeed, the density increased from $\sim 10 \mathrm{~cm}^{-3}$ on Orbit 1 to $\sim 30$ and $45 \mathrm{~cm}^{-3}$ on orbits 3 and 4 , respectively, consistent with ionospheric plasma refilling the eroded plasmasphere during the late recovery phase. Higher-density plasmas result in lower Alfvén speeds and longer pulsation periods. We should note that the waves must exist over a broad range of densities and wave frequencies.

Finally, we tested the hypothesis that the pulsations are generated in close proximity to the plasmapause. The top panels (a) and (b) of Fig. 13 indicate that the pulsations 
(a)

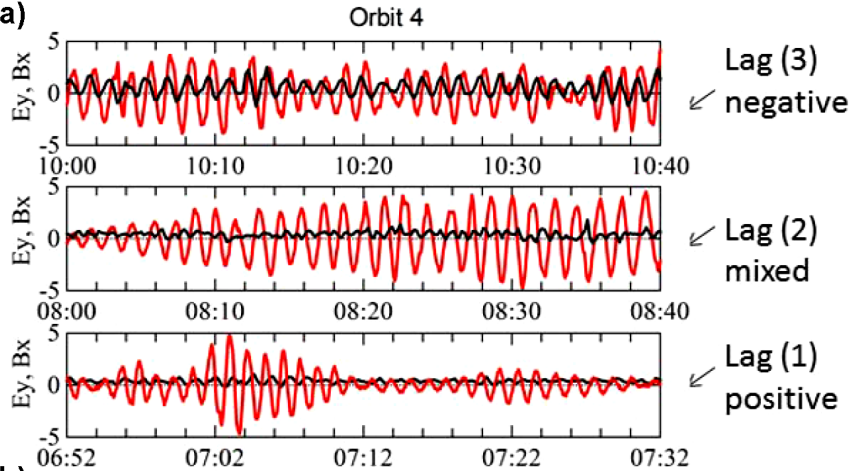

(b)

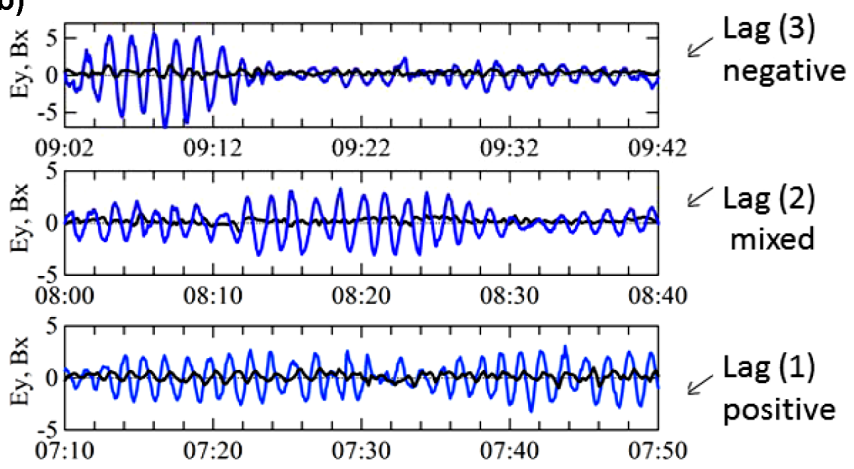

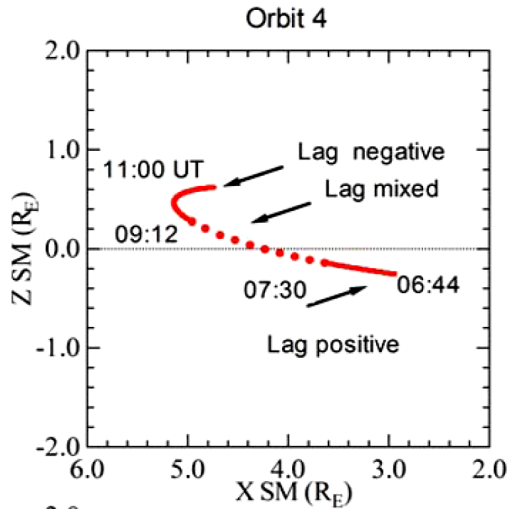

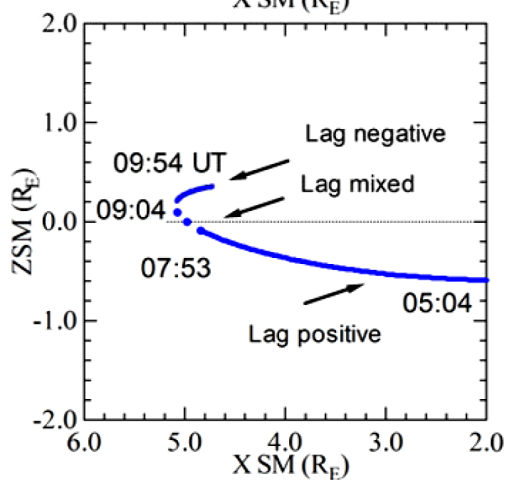

Figure 7. Phase relations between $B_{x}$ (plotted in red and blue) and $E_{y}$ (plotted in black) on Orbit 4 of Van Allen Probes A and B, during 1,2 and 3 intervals (left panel). Locations of the spacecraft relative to the equator (right panel). During the first (third) intervals, $E_{y}$ lags (leads) $B_{x}$ by $90^{\circ}$ when the spacecraft were south (north) of the equator, in agreement with what is expected for second-harmonic waves. Observations during the second intervals when the spacecraft crossed the equator indicate mixed-phase relationships and double-frequency pulsations in $E_{y} . E_{y}$ and $B_{x}$ are displayed in $\mathrm{mV} \mathrm{m}^{-1}$ and $\mathrm{nT}$, respectively.

Orbit 1
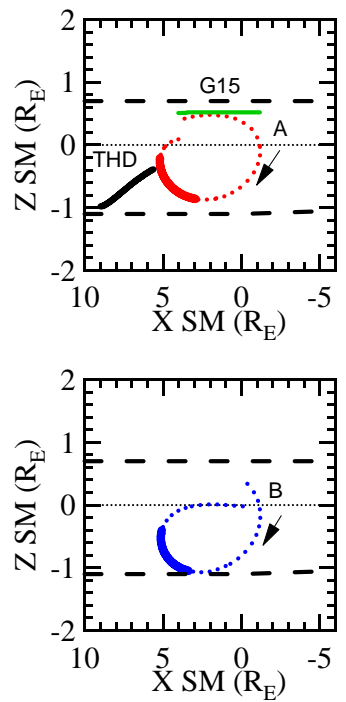

Orbit 2
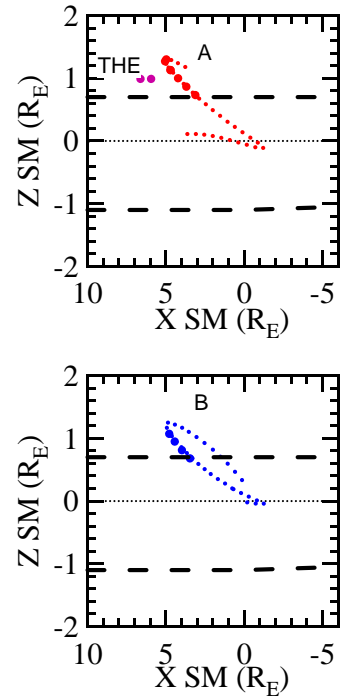

Orbit 3
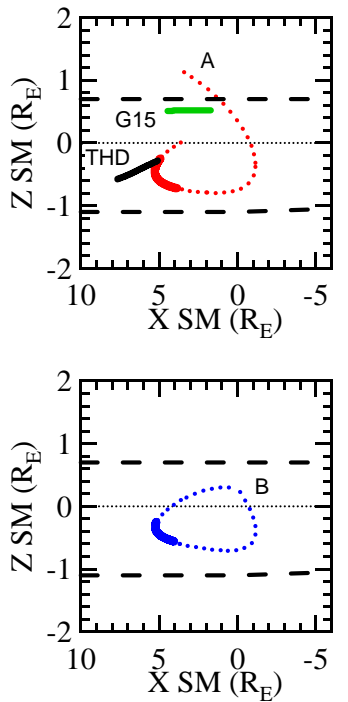

Orbit 4
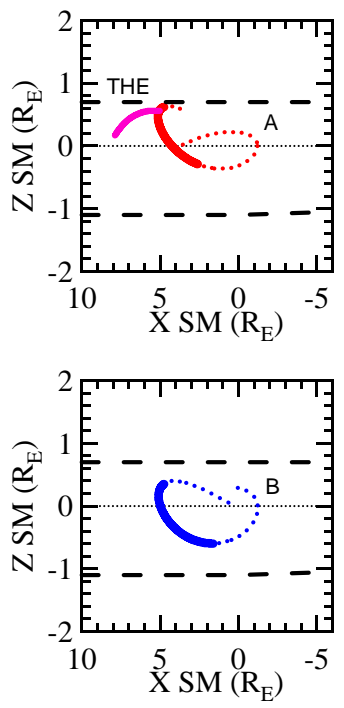

Figure 8. Four successive Van Allen Probes A and B orbits in the SM $X-Z$ plane from 1 00:00 UT on 1 May 2014 to 12:00 UT on 2 May 2014. Colored lines show the locations of the spacecraft where Pc4 pulsations occurred on orbits $1,2,3$ and 4 . The pulsations were limited to $-1.1 R_{\mathrm{E}}<Z_{\mathrm{SM}}<0.7 R_{\mathrm{E}}$ (the region bounded by two dashed black lines). No magnetic field pulsations were observed during the thick dotted line segment in the Van Allen Probes A and B orbits 2 despite the spacecraft being at the appropriate local time and radial distance. 
(a)

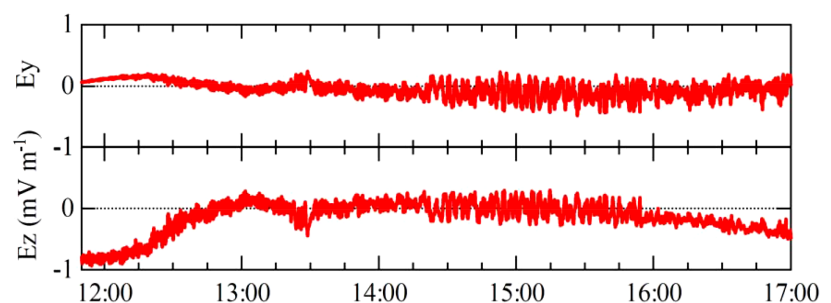

(b)

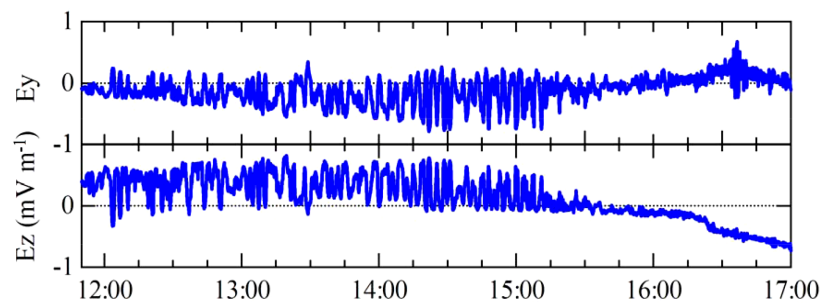

Figure 9. Van Allen Probes A (plotted in red) and B (plotted in blue) observations of electric field from 11:50 to 17:00 UT on 1 May 2014. The electric field pulsations were indeed observed during the second orbit (larger at Van Allen Probe B) though the magnetic field pulsations were absent. We suggest that the spacecraft were near a magnetic field null when at the appropriate local times on this orbit.

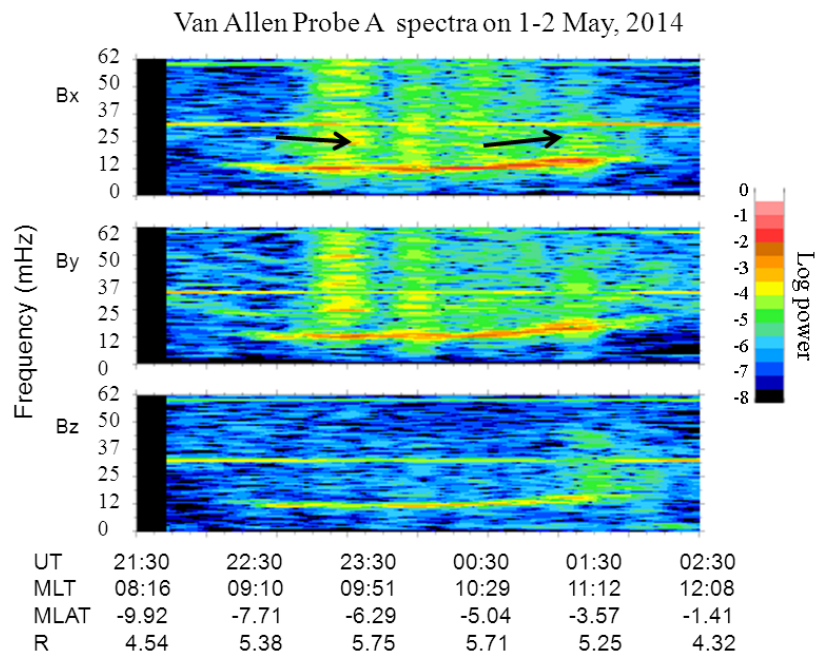

Figure 10. Dynamic spectra calculated for the radial, azimuthal and compressional components of the Van Allen Probe A magnetic field during the Orbit 3 from 21:30 to 02:30 UT on 1-2 May 2014. The location of the satellite is shown in the bottom where $R$ is the radius of Earth, MLAT is magnetic latitude in degrees and MLT is the magnetic local time in hours.

observed on orbits 1 and 3 occurred outside the plasmasphere, in regions with low density and definitely outside any steep plasmapause density gradient. On Orbit 4, some pulsations were observed within or close to a plasmapause that exhibited a very gradual change in density. Since our pulsations were observed over a broad range of $L$ shells beyond (a)

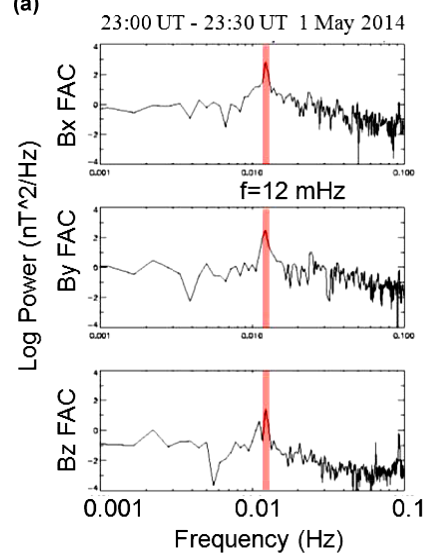

b)
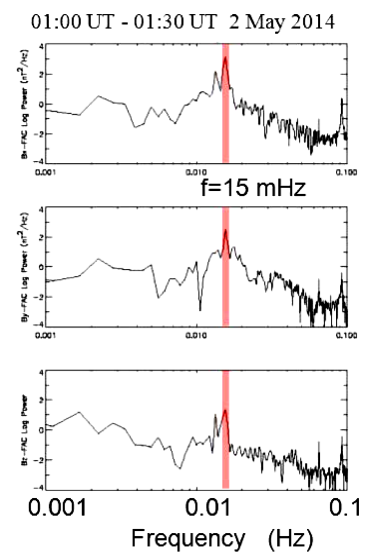

Figure 11. Fourier spectra calculated for the radial, azimuthal and compressional components of the Van Allen Probe A magnetic field in 5 min sliding averaged mean field-aligned coordinates during Orbit 3 from 23:00 to 23:30 UT on 1 May 2014 (a) and from 01:00 to 01:30 UT on 2 May 2014 (b). Vertical lines indicate the frequencies of dominant spectral peaks at 12 and $15 \mathrm{mHz}$. (a)
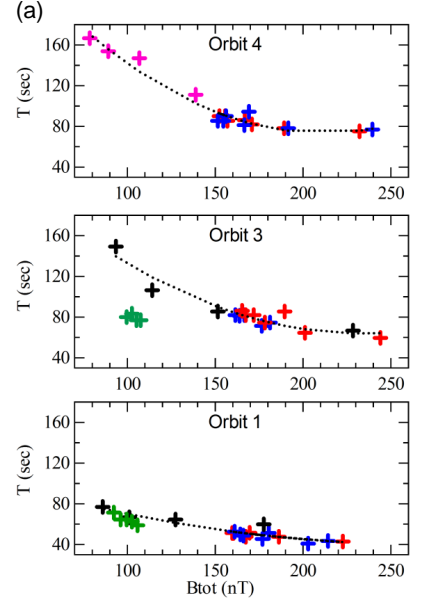

(b)
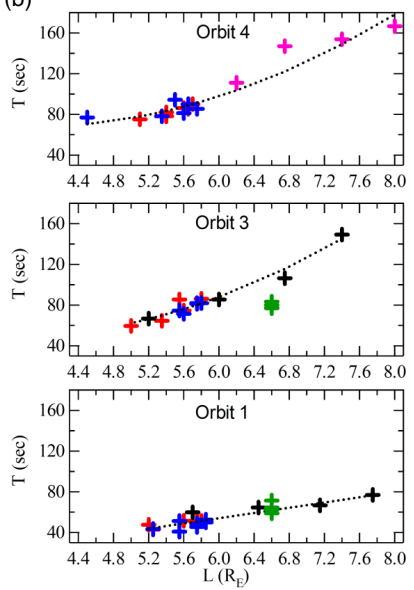

Figure 12. Dominant periods in the spectra of the radial component of the magnetic field observed by Van Allen Probes A (red symbols), B (blue symbols), THEMIS D (black symbols) and E (purple symbols), and GOES 15 (green symbols) as a function of the $30 \mathrm{~min}$ averaged magnetic field strength (a) and $L$ shells (b). Dashed curves represent polynomial fit with three terms.

the plasmapause and did not reach peak amplitudes at the plasmapause, we do not believe that there is any direct relationship between the location of plasmapause and the longlasting Pc4 magnetic field pulsations. But as the mechanism for generating the Pc4 pulsations takes place for a long time, we suppose that the ion population in the ring current remains unstable throughout this time and slow plasmasphere refilling following the storm could contribute to the longevity of the pulsations. There is some evidence that the periods of the pulsations increased during the plasmasphere refilling. Fur- 


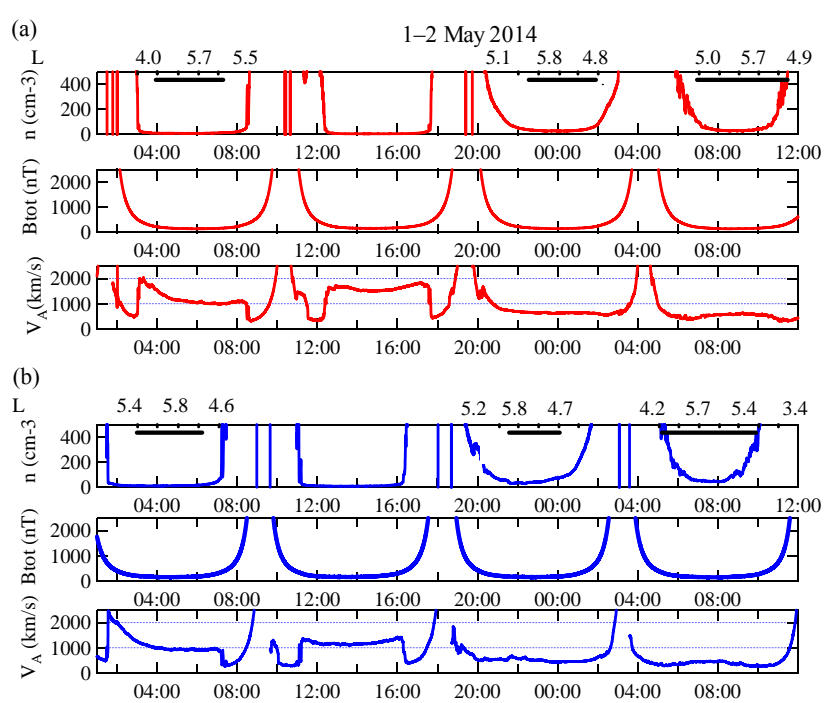

Figure 13. From top to bottom densities, total magnetic field strengths and Alfvén velocities vs. universal time for Van Allen Probes A (plotted in red) and B (plotted in blue) from 01:00 UT on 1 May 2014 to 12:00 UT on 2 May 2014. Solid black bars show intervals when Pc4 pulsations were observed on orbits 1, 3 and 4. Ticks above the panels show spacecraft $L$ values for the corresponding intervals.

ther study comparing model and experimental data will be useful to give a better insight into the structure of poloidal pulsations associated with geomagnetic storm and their possibility to monitor the time evolution of plasma environment in the magnetosphere.

\section{Conclusions}

We studied long-lasting poloidal Pc4 pulsations observed by multi-spacecraft in the dayside magnetosphere. The most striking feature of the Pc4 magnetic field pulsations was their occurrence at similar locations during three of four successive orbits of Van Allen Probes, each $9 \mathrm{~h}$ apart.

They were observed after the main phase of the moderate storm during low geomagnetic activity. The pulsations occurred for a wide range of solar wind conditions and did not appear to respond to any particular solar wind parameter. We found no direct relationship between the plasmapause and the long-lasting Pc4 pulsations.

We showed that the Pc4 pulsations were radially polarized and are second-mode harmonic waves. We found that a phase shift between the azimuthal and radial components of the electric and magnetic fields occurred in the range of $Z_{\mathrm{SM}}$ between 0.30 and $-0.16 R_{\mathrm{E}}$. We demonstrated that the latitudinal nodal extent of the magnetic field pulsations did not exceed $2 R_{\mathrm{E}}$. We suggest that the spacecraft were near a magnetic field null during the second orbit when at the local times where pulsations were observed on previous and successive orbits.
We investigated the spectral structure of the Pc4 pulsations. We found that the dominant periods in the spectra depended on orbit and radial distance. Each spacecraft observed a decrease of the dominant period as it moved to a smaller $L$ (stronger magnetic field strength). On Orbit 1 periods decreased from 76 to $48 \mathrm{~s}$, on Orbit 3 from 149 to $59 \mathrm{~s}$, and on Orbit 4 from 166 to $77 \mathrm{~s}$. The differences between the ranges of periods of the Pc4 pulsations observed by Van Allen Probes on orbits 1, 3 and 4 may originate from external sources such as different interplanetary input parameters or from internal sources such as changes of plasma density distribution along the field lines or at the equator. We showed that higher frequencies occurred at times and locations where Alfvén velocities were greater, i.e., on Orbit 1. There is some evidence that the periods of the pulsations increased during the plasmasphere refilling following the storm. Though the excitation mechanism for these events is not completely clear at this point, we attribute the source of pulsations to bounce resonance oscillations. We will address this statement in a follow-up paper.

\section{Data availability}

Data used in the paper are available publicly at http://cdaweb. gsfc.nasa.gov/istp_public/ (Coordinated Data Analysis Web, 2016).

Acknowledgements. The Van Allen Probes mission is supported by NASA. NASA GSFC's CDAWEB provided Wind and GOES observations, while SSCWEB provided Van Allen Probes EPHEMERIS. Work by G. Korotova at the University of Maryland was supported by grants from NASA NNX15AW86G S01 and NSF AGS1207445. Work performed by M. Engebretson at NASA/GSFC was supported by the Van Allen Probes mission.

The topical editor, Y. Miyoshi, thanks two anonymous referees for help in evaluating this paper.

\section{References}

Anderson, B. J., Engebretson, M. J., Rounds, S. P., Zanetti, L. J., and Potemra, T. A.: A statistical study of Pc 3-5 pulsations observed by the AMPTE/CCE Magnetic Fields Experiment, 1. Occurrence distributions, J. Geophys. Res., 95, 10495-10523, 1990.

Anderson B. J., Potemra, T. A., Zanetti, L. J., and Engebretson, M. J.: Statistical correlations between Pc 3-5 pulsations and solar wind/IMF parameters and geomagnetic indices, in: Physics of Space Plasmas (1990), SPI Conf. Proc. Reprint Ser., vol. 10, edited by: Chang, T., Crew, G. B., and Jasperse, J. R., p. 419, Scientific, Cambridge, MA, USA, 1991.

Arthur, C. W. and McPherron, R. L.: The statistical character of Pc 4 magnetic pulsations at synchronous orbit, J. Geophys. Res., 86, 1325-1334, doi:10.1029/JA086iA03p01325, 1981.

Auster, H. U., Glassmeier, K.-H., Magnes, W., Aydogar, O., Baumjohann, W., Constantinescu, D., Fischer, D., Fornacon, K. 
H., Georgescu, E., Harvey, P., Hillenmaier, O., Kroth, R., Ludlam, M., Narita, Y., Nakamura, R., Okrafka., K., Plaschke, F., Richter, I., Schwarz, H., Stoll, B., Valavanoglou, A., and Wiedemann, M.: The THEMIS Fluxgate Magnetometer, Space Sci. Rev., 141, 235-264, doi:10.1007/s11214-008-9365-9, 2008.

Claudepierre, S. G., Mann, I. R., Takahashi, K., Fennell, J. F., Hudson, M. K., Blake, J. B., Roeder, J. L., Clemmons, J. H., Spence, H. E., Reeves, G. D., Baker, D. N., Funsten, H.O., Friedel, R. H. W., Henderson, M. G., Kletzing, C. A., Kurth, W. S., MacDowall., R. J., Smith, C. W., and Wygant, R.: Van Allen Probes Observation of Localized Drift-Resonance Between Poloidal Mode Ultra-low Frequency Waves and $60 \mathrm{keV}$ Electrons, Geophys. Res. Lett., 40, 4491-4497, doi:10.1002/grl.50901, 2013.

Coordinated Data Analysis Web: Public data from current space physics missions, available at: http://cdaweb.gsfc.nasa.gov/istp_ public/, last access: 10 November 2016.

Cramm, R., Glassmeier, K. H., Othmer, C., Fornacon, K. H., Auster, H. U., Baumjohann, W., and Georgescu, E.: A case study of a radially polarized $\mathrm{Pc} 4$ event observed by the Equator-S satellite, Ann. Geophys., 18, 411-415, doi:10.1007/s00585-000-0411-5, 2000.

Cummings, W. D., O’Sullivan, R. J., and Coleman Jr., P. J.: Standing Alfvén waves in the magnetosphere, J. Geophys. Res., 74, 778793, 1969.

Dai, L., Takahashi, K., Wygant, Chen, L., Bonnell, J., Cattell, C. A., Thaller, S., Kletzing, C., Smith, C. W., MacDowall, R. J, Baker, D. N., Blake, Fennell, J., Claudepierre, S., Funsten, H. O., Reeves, G. D., and Spence, H. E.: Excitation of poloidal standing Alfven waves through drift resonance wave-particle interaction, Geophys. Res. Lett., 40, 4127-4132, doi:10.1002/grl.50800, 2013.

Dai, L., Takahashi, K., Lysak, R., Wang, C., Wygant, J. R., Kletzing, C., Bonnell, J., Cattell, C. A., Smith, C. W., MacDowall, R. J., Thaller, S., Breneman, A., Tang, X., Tao, X., and Chen, L.: Storm time occurrence and spatial distribution of Pc4 poloidal ULF waves in the inner magnetosphere: A Van Allen Probes statistical study, J. Geophys. Res.-Space, 120, 47484762, doi:10.1002/2015JA021134, 2015.

Engebretson, M., Erickson, K. N., Strangeway, R. J., Klumpar, D. M., Fuselier, S. A., Zanetti, L. J., and Potemra, T. A.: The spatial extent of radial magnetic pulsation events observed in the dayside near synchronous orbit, J. Geophys. Res., 97, 13741-13758, 1992.

Engebretson, M. J., Posch, J. L., Wygant, J. R., Kletzing, C. A., Lessard, M. R., Huang, C.-L., Spence, H. E., Smith, C. W., Singer, H. J., Omura, Y., Horne, R. B., Reeves, G. D., Baker, D. N., Gkioulidou, M., Oksavik, K., Mann, I. R., Raita,T., and Shiokawa, K.: Van Allen probes, NOAA, GOES, and ground observations of an intense EMIC wave event extending over $12 \mathrm{~h}$ in magnetic local time, J. Geophys. Res.-Space, 120, 5465-5488, doi:10.1002/2015JA021227, 2015.

Eriksson, P. T. I., Blomberg, L. G., Walker, A. D. M., and Glassmeier, K.-H.: Poloidal ULF oscillations in the dayside magnetosphere: a Cluster study, Ann. Geophys., 23, 2679-2686, doi:10.5194/angeo-23-2679-2005, 2005.

Higbie, P. R., Baker, D. N., Zwickl, R. D., Bellian, R. D., Asbridge, J. R., Fennell, J. F., Wilken, B., and Arthur, C. W.: The Global Pc 5 Event of November 14-15, 1979, J. Geophys. Res., 87, 2337$2345,1982$.
Hughes, W. J., Southwood, D. J., Mauk, B., McPherron, R. L., and Barfield, J. N.: Alfven waves generated by an inverted plasma energy distribution, Nature, 275, 43-45, 1978.

Hughes, W. J., McPherron, R. L., Barfield, J. N., and Mauk, B. H.: A compressional Pc 4 pulsation observed by three satellites in geostationary orbit near local midnight, Planet. Space Sci., 27, 821-840, 1979.

Kletzing, C. A., Kurth, W. S., Acuna, M., MacDowall, R. J, Torbert, R. B., Averkamp, T., Bodet, D., Bounds, S. R., Chutter, M., Connerney, J., Crawford, D., Dolan, J. S., Dvorsky R., Hospodarsky, G. B., Howard, J., Jordanova, V., Johnson, R. A., Kirchner, D. L., Mokrzycki, B., Needell, G., Odom, J., Mark, D., Pfaff Jr., Phillips, J. R. , Piker, C.W., Remington, S. L., Rowland, D., Santolik, O., Schnurr, R., Sheppard, D., Smith, C. W., Thorne, R. M., and Tyler, J. J.: The Electric and Magnetic Field Instrument Suite and Integrated Science (EMFISIS) on RBSP, Space Sci. Rev., 179, 127-181, doi:10.1007/s11214-013-9993-6, 2013.

Kokubun, S., Erickson, K. N., Fritz, T. A., and McPherron, R. L.: Local time asymmetry of Pc 4-5 pulsations and associated particle modulations at synchronous orbit, J. Geophys. Res., 94, 6607-6625, 1989.

Korotova, G. I., Sibeck, D. G., Angelopoulos, V., and Walsh, W.: Themis observations of compressional poloidal pulsations in the dawnside magnetosphere: a case study, J. Geophys. Res., 118, 7665-7673, doi:10.1002/2013JA019360, 2013.

Korotova, G. I., Sibeck, D. G., Tahakashi, K., Dai, L., Spence, H. E., Kletzing, C. A., Wygant, J. R., Manweiler, J. W., Moya, P. S., Hwang, K.-J., and Redmon, R. J.: Van Allen Probe observations of drift-bounce resonances with Pc 4 pulsations and waveparticle interactions in the pre-midnight inner magnetosphere, Ann. Geophys., 33, 955-964, doi:10.5194/angeo-33-955-2015, 2015.

Lepping, R. P., Acuna, M. H., Burlaga, L. F., Farrell, W. M., Slavin, J. A., Schatfen, K. H., Mariani, E., Ness, N. E., Neubauer, E. M., Whang, Y. C., Byrnes J. B., Kennon R. S., Panetta, P. V., Scheifele J., and Worley, E. M.: The WIND Magnetic field investigation, Space Sci. Rev., 71, 207-229, 1995.

Lin, N. G., Cahill, L. J., Engebretson, M. J., Sugiura, M., and Arnoldy, R. L.: Dayside pulsation events near the plasmapause, Planet. Space Sci., 34, 155-181, 1986.

Mauk, B. H., Fox, N. J., Kanekal, S. G., Kessel, R. L., Sibeck, D. G., and Ukhorskiy, A.: Science objectives and rationale for the radiation belt storm probes mission, Space Sci. Rev., 179, 3-27, doi:10.1007/s11214-012-9908-y, 2012.

Motoba, T., Takahashi, K., Ukhorskiy, A. A., Gkioulidou, M., Mitchell, D. G., Lanzerotti, L. J., Mauk, B., Korotova, G. I., Donovan, E., Wygant, J. R., Kletzing, C. A., Kurth, W., and Blake, J. B.: Link between pre-midnight second harmonic poloidal waves and auroral undulations: Conjugate observations with a Van Allen Probes spacecraft and a THEMIS GBO all-sky camera, J. Geophys. Res., 120, 1814-1831, doi:10.1002/2014JA020863, 2015.

Nagano, H. and Araki, T.: Long-duration Pc5 pulsations observed by geostationary satellites, Geophys. Res. Lett., 10, 908-911, 1983.

Ogilvie, K. W., Chornay, D. J., Fritzenreiter, R. J., Hunsaker, F., Keller, J., Lobell, J., Miller, G., Scudder, J. D., Sittler, E. C., Torbert, R. B., Bodet, D., and Needell, G.: SWE, a comprehensive 
plasma instrument for the WIND spacecraft, Space Sci. Rev., 71, 55-77, doi:10.1007/BF00751326, 1995.

Orr, D. and Matthew, J. A. D.: The variation of geomagetic micropulsation periods with latitude and the plasmapause, Planet. Space Sci., 19, 897-905, 1971.

Poulter, E. M., Allan W., and Bailey G. J.: ULF pulsation eigenperiods within the plasmasphere, Planet. Space Sci., 36, 185-196, 1988.

Rostoker, G., Lam, H.-L., Olson, J. V.: Pc4 giant pulsations in the morning sector, J. Geophys. Res., 84, 5153-5166, doi:10.1029/JA084iA09p05153, 1979.

Sarris, T. E., Loto'aniu, T. M., Li, X., and Singer, H. J.: Observations at geosynchronous orbit of a persistent Pc5 geomagnetic pulsation and energetic electron flux modulations, Ann. Geophys., 25, 1653-1667, doi:10.5194/angeo-25-1653-2007, 2007.

Schäfer, S., Glassmeier, K. H., Eriksson, P. T. I., Pierrard, V., Fornaçon, K. H., and Blomberg, L. G.: Spatial and temporal characteristics of poloidal waves in the terrestrial plasmasphere: a CLUSTER case study, Ann. Geophys., 25, 1011-1024, doi:10.5194/angeo-25-1011-2007, 2007.

Schäfer, S., Glassmeier, K. H., Eriksson, P. T. I., Mager, P. N., Pierrard, V., Fornaçon, K. H., and Blomberg, L. G.: Spatio-temporal structure of a poloidal Alfvén wave detected by Cluster adjacent to the dayside plasmapause, Ann. Geophys., 26, 1805-1817, doi:10.5194/angeo-26-1805-2008, 2008.

Singer, H. J., Russell, C. T., Kivelson, M. G., Fritz, T. A., and Lennartsson, W.: Satellite observations of the spatial extent and structure of Pc 3, 4, 5 pulsations near the magnetospheric equator, J. Geophys. Res., 6, 889-892, 1979.

Singer, H. J., Hughes, W. J., and Russell, C. T.: Standing hydromagnetic waves observed by ISEE 1 and 2: Radial extent and harmonic, J. Geophys. Res., 87, 3519-3529, doi:10.1029/JA087iA05p03519, 1982.

Singer, H. J., Matheson, L., Grubb, R., Newman, A., and Bouwer, S. D.: Monitoring space weather with the GOES magnetometers, in: SPIE Conference Proceedings, vol. 2812, edited by: Washwell, E. R., 299-308, GOES-8 and Beyond SPIE, Bellingham, WA, USA, 1996.

Southwood, D. J.: A general approach to low-frequency instability in the ring current plasma, J. Geophys. Res., 81, 3340-3348, 1976.
Southwood, D. J. and Kivelson, M. G.: Charged particle behavior in low-frequency geomagnetic pulsations, 2. Graphical approach, J. Geophys. Res., 87, 1707-1710, 1982.

Sugiura, M. and Wilson, C. R.: Oscillation of the geomagnetic field lines and associated magnetic perturbations at conjugate points, J. Geophys. Res., 69, 1211-1216, 1964.

Takahashi, K. and McPherron, R. L.: Standing hydromagnetic oscillations in the magnetosphere, Planet. Space Sci., 32, 1343-1359, 1984.

Takahashi, K., Higbie, P. R., and Baker, D. N.: Energetic electron flux pulsations observed at geostationary orbit: Relation to magnetic pulsations, J. Geophys. Res., 90, 8308-8318, doi:10.1029/JA090iA09p08308, 1985.

Takahashi, K., Zanetti, L. J., Potemra, T. A., and Acuña, M. H.: A model for the harmonic of compressional Pc 5 waves, Geophys. Res. Lett., 14, 363-366, 1987.

Takahashi, K., Hartinger, M. D., Angelopoulos, V., Glassmeier, K.H., and Singer, H. J.: Multispacecraft observations of fundamental poloidal waves without ground magnetic signatures, J. Geophys. Res.-Space, 118, 4319-4334, doi:10.1002/jgra.50405, 2013.

Wild, J. A., Yeoman, T. K., and Waters, C. L.: Revised time-of-flight calculations for high-latitude geomagnetic pulsations using a realistic magnetospheric magnetic field model, J. Geophys. Res., 110, A11206, doi:10.1029/2004JA010964, 2005.

Wygant, J. R., Bonnell, J. W., Goetz, K., Ergun, R. E., Mozer, F. S., Bale, S. D., Ludlam, M., Turin, P., Harvey, P. R., Hochmann, R., Harps, K., Dalton, G., McCauley, J., Rachelson, W., Gordon, D., Donakowski, B., Shultz, C., Smith, C., Diaz-Aguado, M., Fischer, J., Heavner, S., Berg, P., Malaspina, D. M., Bolton, M. K., Hudson, M., Strangeway, R. J., Baker, D. N., Li, X., Albert, J., Foster, J. C., Chaston, C. C., Mann, I., Donovan, E., Cully, C. M., Cattell, C. A., Krasnoselskikh, V., Kersten, K., Breneman, A., and Tao, J. B.: The electric field and waves instruments on the radiation belt storm probes mission, Space Sci. Rev., 179, 183-220, doi:10.1007/s11214-013-0013-7, 2013. 